\title{
Über das Mögliche urteilen. Urteilsfindung in Kriegswirtschaftsverfahren am Sondergericht Wien
}

\author{
Gabriele Hackl
}

\section{Abstract}

The National Socialist regime produced systematic insecurity in order to induce new procedures, legal norms and case law. In this paper, Gabriele Hackl uses the example of war economy proceedings before the Vienna Special Court to inquire into the role of the National Socialist securitisation strategy in judicial practice in the context of criminal law during the National Socialist era. The author interprets court proceedings as processes of differentiating between (groups of) people, which were justified on grounds of protection and security. What emerges as the reference point of these arguments is a Volksgemeinschaft (people's community) that was perceived to be under constant threat. The - often complete - removal of (potentially) dangerous delinquents from this community became a security priority. To identify the (potentially) dangerous, jurists applied various criteria in assessing the deed and the person in question, such as the magnitude of the deed, the accused's criminal record, gender-conforming behaviour, "race", social background or the person's health or (dis)ability to perform. It was not uncommon for a defendant or a defendant's attorney to invoke these criteria in the trial in the hope of obtaining a more lenient sentence. Drawing on several cases, this contribution shows that the significance of these arguments in reaching the verdict was highly situational. What becomes clear is that both the prosecutors and the judges of the Vienna Special Court, not to mention the National Socialist judicial apparatus as a whole, were deeply involved in processes of securitisation. And the judgements they rendered did not only address the actual but the possible.

\section{Einleitung: Der nationalsozialistische Justizapparat als Sicherheitsorgan}

Die erste Assoziation, wenn es um Institutionen des Sicherheitsbereichs geht, sind oftmals die Exekutivorgane: Polizei, Militär und Geheimdienste sollen für „Ruhe und Ordnung“ sorgen und die physische Sicherheit der Staatsbürger*innen gewährleisten. Auch Politiker*innen als Repräsentant"innen der Staatsgewalt stehen im Rampenlicht der Sicherheitsfor- 
schung. Neben diesen klassischen Sicherheitsakteur*innen ${ }^{1}$ rückt in der Literatur der sog. Pariser Schule, die ein breiteres Verständnis des Sicherheitsbereichs und von Sicherheitsakteur*innen geschaffen hat, auch die Justiz in den Blick des Fachdiskurses.

Jurist"innen nehmen neben den Gewaltexpert"innen, Verhandler*innen und Wissensexpert"innen eine besondere Stellung ein, da sie zum einen über fachspezifisches Wissen verfügen, auf dessen Basis sie Handlungsempfehlungen aussprechen können, zum anderen als Staatsanwält"innen und Richter*innen aber auch selbst Entscheidungen von erheblicher Tragweite treffen können (vgl. Reusch et al. 2018, S.95ff.). In Gerichtsverhandlungen verhandeln Jurist*innen wortwörtlich über Bedrohungen und angemessene Sicherheitsmaßnahmen. Durch ihre alltäglichen Praktiken und Routinen stellen sie ständig (Un-)Sicherheit her (vgl. Westermeier/Carl 2018, S. 18). Sie untermauern Entscheidungen rechtlich und verschaffen damit Schutzmaßnahmen Legitimität und dauerhafte Geltung (vgl. Reusch et al. 2018, S. 99).

Auch in der NS-Ära nahmen Juristen ${ }^{2}$ - wie die übrigen staatstragenden Akteure - aktiv an Versicherheitlichungsprozessen ${ }^{3}$ teil. Schon von Beginn an machten sie sich daran, Konsens über diffuse Bedrohungen und rechtliche Grundlagen für dagegen zu ergreifende Schutzmaßnahmen zu schaffen. Die Argumentation der Sicherung durch Schutz ist aus den nationalsozialistischen Rechtsnormen nicht wegzudenken. Das NS-Regime legitimierte seine Gesetzgebung und seine Rechtsprechungspraxis mit der Antizipation von Bedrohungen. Unsicherheiten wurden gezielt geschaffen, um die Implementierung neuer Normen, Verfahrens- und Rechtsprechungspraktiken zu provozieren (vgl. Reusch et al. 2018, S. 96). Als gefährdet dargestellt wurden dabei in erster Linie das „deutsche Volk“, seine „Reinheit“ und Gesundheit, seine Lebensgrundlage (ob bezogen auf den „Lebensraum" oder die Versorgung) und - nach Kriegsbeginn - seine Wehrfähigkeit. Das NS-Regime gab nicht nur die Garantie ab, vor jenen zu schützen, die es dezidiert kriminalisierte, sondern auch vor all jenen, die es als

1 Sicherheitsakteur*innen werden im Folgenden verstanden als Akteur*innen im Feld der Sicherheit, die durch ihr verbales und nonverbales Agieren, durch Diskurse und Praktiken, den Prozess der Versicherheitlichung gestalten (vgl. Westermeier/Carl 2018).

2 Damals handelte es sich noch stärker als heutzutage um ein männliches Betätigungsfeld.

3 Versicherheitlichung wird nach Balzacq (2010, S. XIII) definiert als ein Prozess der Produktion, Verbreitung und Rezeption von Bedrohungen des Sicherheitsempfindens. Versicherheitlichung ist damit die Konstruktion von (Un-)Sicherheit. 
„Schädlinge“ und „Parasiten“ kennzeichnete. Die Bedrohungsszenarien kreisten um viele verschiedene Personen(gruppen): Kriminelle, ethnische und religiöse Minderheiten, Kranke, Arme und andere sozial Marginalisierte.

Das NS-Regime setzte auf die Angst vor angeborener „Minderwertigkeit“ und „fremdem Blut“. Einschlägige Diskurse waren seit dem 19. Jahrhundert mit jenen über Kriminalität und anderes „asoziales“ Verhalten dicht verwoben (vgl. Wetzell 2000). Durch Biologisierung wurden diese verschiedenen Dimensionen von sozialer und biologischer (und politischer) Gefahr untrennbar miteinander verknüpft. Aufgabe der Exekutivorgane $^{4}$ und des Justizapparats war es, der „Volksgemeinschaft“5 gegen all diese Gefahren Schutz zu bieten und die politische und gesellschaftliche Entwicklung in die gewünschten Bahnen zu lenken. Die Staatsanwaltschaften und Gerichte dienten der Kontrolle unerwünschten Verhaltens ob es durch (neue) Gesetze und Verordnungen bereits kriminalisiert war oder erst durch „kreative“ Rechtsauslegung und Rechtsbeugung ad hoc kriminalisiert werden musste. Sie schufen, wie die Exekutive auch, als „Instrumente der Kontrolle und Repression [...] erst die Verbrechen, die zu kontrollieren sie beabsichtig[t]en" (Kunze 2005, S. 16). Rechtliche Neuerungen, wie die Abschaffung des Analogieverbots und gleichzeitige Einführung der Generalklausel des „gesunden Volksempfindens" ${ }^{\text {"6 }}$ oder das

4 Die sogenannte Vorbeugehaft und die sogenannte Schutzhaft lagen im Zuständigkeitsbereich von Polizei und SS. Zur Verfolgung von „Berufsverbrechern“ und „Berufsverbrecherinnen“ sowie „Gewohnheitsverbrechern“ und „Gewohnheitsverbrecherinnen" durch Polizeibehörden in Österreich vgl. Hörath 2017 und Kranebitter 2018.

5 Die „Volksgemeinschaft“ wird als eine gedachte, handlungsrelevante Ordnung definiert. Insoweit, als sie Handlungen zur Folge hatte, war sie auch eine „gelebte Ordnung“. Forschungsarbeiten zu In- und Exklusion und „Volksgemeinschaft“ siehe zum Beispiel Bajohr/Wildt 2009.

6 Das Analogieverbot sieht vor, dass eine Tat nur gerichtlich geahndet werden kann, wenn im Strafrecht ein entsprechender Tatbestand normiert ist. Durch die Abschaffung dieses Verbots mit der Neufassung des $\$ 2$ RStGB im Jahr 1935 konnte jede Tat „die nach dem Grundgedanken eines Strafgesetzes und nach gesundem Volksempfinden Bestrafung verdient" durch Heranziehung eines bestehenden Gesetzes abgestraft werden, auch wenn es die Tat nicht dezidiert erfasste. Vgl. „Gesetz zur Änderung des Strafgesetzbuchs“ vom 28.6.1935. RGBl. I, S. 839-843. Das österreichische Strafgesetzbuch wurde nach dem „Anschluss“ nicht entsprechend abgeändert, die Sondergerichte in den Alpen- und Donaureichsgauen wandten aber beide Strafbücher an, so auch $\$ 2$ RStGB. 
Argument des „Schutzes der Volksgemeinschaft"7, begründeten die Aussonderung der Schutzunwürdigen und Gefährlichen. ${ }^{8}$

Im Folgenden soll am Beispiel von Kriegswirtschaftsverfahren ${ }^{9}$ am Sondergericht Wien gezeigt werden, wie die Richter und Staatsanwälte bei der Identifikation und Bestrafung der Gefährlichen in Gerichtsprozessen vorgingen, das heißt, wann und wem gegenüber sie Schutz- und Sicherheitsargumente anwandten und welche Bedeutung sie tat- oder personenbezogenen Merkmalen - die unter die heute gängigen Differenzkategorien (vgl. Winker/Degele 2010) zu fassen sind - zumaßen. Deutlich wird, dass die als ständig bedroht konzipierte „Volksgemeinschaft“ als Referenzpunkt bei der Versicherheitlichung eine maßgebliche Rolle spielte.

\section{Das Sondergericht Wien und die bedrohte „Volksgemeinschaft"}

Die Sondergerichte waren zuständig für einen Großteil der von den Nationalsozialist*innen erlassenen Gesetze und Verordnungen. Sie konstituierten die nationalsozialistische Sicherheitskultur anhand effektiver Bedrohungsszenarien durch $\mathrm{Ab}$ - und Ausgrenzung, durch die Unterscheidung von Freund und Feind (vgl. Conze 2018, S. 80). Als außerordentliche Gerichte, die drakonische Strafen verhängten, gerieten sie früh in den Blick zeithistorischer Forschung. ${ }^{10}$

7 Beispielsweise im „Gesetz zur Änderung des Reichstrafgesetzbuches“ vom 4.9.1941 („Änderungsgesetz“, RGB1. I, $\mathbb{1} 1$, S. 549): „Der gefährliche Gewohnheitsverbrecher ( $\$ 20$ a des Strafgesetzbuchs) und der Sittlichkeitsverbrecher ( $\mathbb{S} 176$ bis 178 des Strafgesetzbuchs) verfallen der Todesstrafe, wenn der Schutz der Volksgemeinschaft oder das Bedürfnis nach gerechter Sühne es erfordern.“

8 Wie Michael Wagner-Kern bereits formulierte: „so orientierte sich die NS-Gesetzgebung von Beginn an an einer Zwecksetzung, die mittels eines unklaren Gefährdungsgedankens den stets ebenso nebulös bleibenden ,Schutz der Volksgemeinschaft' zur [sic! ] einer strafrechtlichen Leitlinie erklärte“ (2016, S. 69).

9 Das primäre Kriterium bei der Auswahl der Fallbeispiele war, neben der Sichtbarkeit der Sicherheits- und Schutzargumente, die Möglichkeit, auch die Argumentationslinie der Beschuldigten nachvollziehen zu können. Aus diesem Grund beschränken sich die beschriebenen Fälle auf die Jahre 1943 und 1944.

10 Auf eine Aufzählung der zahlreichen Publikationen zu Sondergerichten des „Altreichs“ wird hier verzichtet. Festzuhalten ist aber, dass sich nur wenige der Installation und Praxis der Sondergerichte in den angegliederten oder besetzten Gebieten widmen, insbesondere der Oberlandesgerichtsbezirke Breslau (poln. Wrocław), Danzig (poln. Gdańsk), Graz, Leitmeritz (tschech. Litoměřice), Posen (poln. Poznań), Stettin (poln. Szczecin) und Wien. 
Das Sondergericht Wien stellte das größte politische Schnellgericht des NS-Regimes auf ehemals österreichischem Boden dar. ${ }^{11}$ Als Sondergericht verhandelte es über viele verschiedene Delikte, die politische, politisierte oder auch als unpolitisch geltende sein konnten. Es urteilte über kritische Äußerungen genauso wie über Schwarzschlachtungen, Plünderungen oder „Rassenschande“. Nach heutigem Wissensstand waren die häufigsten verhandelten Delikte Vergehen, die nach dem „Gesetz gegen heimtückische Angriffe auf Staat und Partei und zum Schutz der Parteiuniformen" vom 20. Dezember 1934 (RGBl. I, S. 1269) oder den $\$ \$ 130,134 \mathrm{a}$ und 134b Reichsstrafgesetzbuch (RStGB) ${ }^{12}$ abgestraft wurden, sowie sogenannte Kriegswirtschaftsverbrechen. Während das „Heimtückegesetz“ die nationalsozialistische Bewegung und ihre Organisationen vor Angriffen unterschiedlicher Art schützen sollte, stellte die „Kriegswirtschaftsverordnung“ (RGBl. I 1939, S. 1609-1613) sich als Maßnahme der Sicherung der Ökonomie und der Versorgung der „Volksgenossen“ und „Volksgenossinnen“ dar. Die Verordnung stellte das Beiseiteschaffen aller bewirtschafteten Güter (und von Geldwerten) unter Strafe. Vorgesehen waren Gefängnis- und Zuchthausstrafen, in besonders schweren Fällen aber auch die Todesstrafe. Es sind vor allem diese schweren Fälle, bei denen die Todesstrafe drohte, bei denen auch Schutz- und Sicherheitsargumente im Urteil angeführt werden.

Der nationalsozialistische Sicherheitsdiskurs traf in den juristischen Kreisen Österreichs - bereits vor dem „Anschluss“ und der darauffolgenden „Säuberung“ des Justizpersonals - auf Rezipienten, die sich bereit zeigten, ihn mitzutragen und konsequent Handlungsfolgen abzuleiten. ${ }^{13}$

$11 \mathrm{Ab} 1938$ vorerst als Spezieller Senat an den Oberlandesgerichten eingerichtet, waren die Sondergerichte ab Frühjahr 1940 - wie im „Altreich“ auch - an den Landgerichten tätig. Vgl. „Verordnung über die Erweiterung der Zuständigkeiten der Sondergerichte" vom 20.11.1938 (GBlÖ. Nr. 614/1938) und „Verordnung über die Zuständigkeit der Strafgerichte, die Sondergerichte und sonstige strafverfahrensrechtliche Vorschriften“ vom 21.2.1940 (RGBl. I, 405-411). Im Oberlandesgerichtsbezirk Wien befanden sich außerdem die Sondergerichte Krems, St. Pölten und Znaim, die 1941 und 1942 in das Sondergericht Wien integriert wurden.

$12 \$ 134 \mathrm{a}$ "schützte“ das Reich, die Länder, die Verfassung, staatliche Symbole (etwa Flaggen oder Farben) und die deutsche Wehrmacht vor Beschimpfungen oder sogenannter böswilliger Verächtlichmachung. $\$ 134 \mathrm{~b}$ legte dasselbe für die NSDAP, ihre Gliederungen, Hoheitszeichen, Standarten und Fahnen, Abzeichen und Auszeichnungen fest. $\$ 130$ RStGB ahndete den sogenannten Kanzelmissbrauch, also unerwünschte Aussagen Geistlicher.

13 Zur ideologischen Ausrichtung der (Strafrechts-)Juristen der 1. Republik Österreich gibt es zwar bis dato keine umfassende quantitative Erhebung, jedoch 
Die Juristen der Staatsanwaltschaft und des Sondergerichts am Landgericht Wien zogen bereitwillig eine Grenze zwischen den dem NS-Regime wertvoll und schützenswert erscheinenden „Volksgenossen“ und „Volksgenossinnen“, und der heterogenen Gruppe der Unerwünschten, die im Nationalsozialismus eine zu bewältigende (potenzielle) Gefahr darstellten. Sie waren dabei in ihrer Wahrnehmung nicht nur geprägt vom politischen und kriminologischen, sondern auch vom medizinischen Fachdiskurs. Unterschiedliche Dimensionen sozialer Ungleichheit, wie „Rasse“/,Volkstum", Klasse, Geschlecht und Körper/Leistungsfähigkeit ${ }^{14}$, wurden von den Juristen in ihrer Abwägung von Gefahr und Nutzen für die „Volksgemeinschaft" berücksichtigt.

Die „Volksgemeinschaft“-Utopie der Nationalsozialist"innen war unter dem Vorzeichen der Bedrohung konzipiert worden: Die angestrebte „Volksgemeinschaft“ war immer auch gefährdet. Die Bedrohung auf eine Idealvorstellung auszurichten, hatte den Vorteil, dass der ausgerufene Ausnahmezustand ${ }^{15}$ zur politischen und gesellschaftlichen Umgestaltung so lange bestehen konnte, wie diejenigen, die über die Deutungshoheit verfügten, das Ideal als noch nicht erreicht bezeichneten (vgl. Landwehr 2008, S. 63). So konnte das NS-Regime radikales Vorgehen begründen und effektiv gegen Gegner“innen vorgehen. Die „Volksgenossen“ und „Volksgenossinnen" wiederum veranlasste die wahrgenommene Bedrohung zur verstärkten Integration. Die „Volksgemeinschaft“ diente der Verhaltensorientierung und sollte den sicheren Handlungsrahmen darstellen (vgl. Conze 2018, S. 156).

Die Sondergerichte sollten an der Verwirklichung dieses Ideals maßgeblich mitwirken. Deshalb urteilten sie nicht unbedingt über das Tatsächliche, sondern über das Mögliche: Sie hatten entsprechend ihrem (gesell-

genügend Hinweise auf eine generelle Nähe zum großdeutschen oder nationalsozialistischen Milieu (vgl. Wedrac 2017).

14 Körper als Differenzkategorie wird im Folgenden verstanden als Marker der physischen und psychischen Gesundheit, die der Bewertung der "Minderwertigkeit“ oder „Entartung“ einer Person zugrunde lag und sich stark auf deren Leistungsfähigkeit bezog.

15 Der Begriff des Ausnahmezustands ist unumstößlich mit dem Staats- und Völkerrechtler Carl Schmitt verbunden, der in seinem erstmals 1922 erschienenen Werk „Politische Theologie“ eine Definition des Ausnahmefalls wie auch des Souveräns liefert und in dem Zusammenhang auch von Gefährdung und Sicherheit spricht. Ersterer ist bei Schmitt der „Fall äußerster Not, Gefährdung der Existenz des Staates“, Letzterer ist jener, der „im Konfliktsfall entscheidet, worin das öffentliche oder staatliche Interesse, die öffentliche Sicherheit und Ordnung, le salut public usw. besteht." (Schmitt 2015, S. 13). 
schafts-)politischen Auftrag Gefahren zu antizipieren und als gefährlich identifizierte Personen (vorbeugend) auszuschalten. In diesem Sinne muss die Rolle der Juristen des NS-Regimes als extreme Involvierung in Versicherheitlichungsprozesse verstanden werden.

\section{Projektive Beurteilungen von Gefährlichkeit}

Am offensichtlichsten argumentierten die involvierten Juristen mit dem Schutz der „Volksgemeinschaft“ in Fällen, in denen sie nicht nur nach der „Kriegswirtschaftsverordnung“ anklagten bzw. verurteilten, sondern die Täter"innen zusätzlich als „Gewohnheitsverbrecher“ oder „Gewohnheitsverbrecherin" einstuften. ${ }^{16}$ Meist handelte es sich bei diesen Fällen um (Einbruchs-)Diebstähle, die bewirtschaftete Güter betrafen und teilweise während der kriegsbedingten Verdunkelung begangen wurden. So etwa im Fall des Wiener Hilfsarbeiters Josef Kiesling, ${ }^{17}$ der mit mehreren Komplizen Süßungsmittel (Saccharin und Kandisette) aus einer Fabrik gestohlen hatte.

Die Strafsache gegen den Erstangeklagten und die anderen Mittäter sollte im vereinfachten Verfahren vor dem Landgericht Wien behandelt werden. Das Verfahren gegen Josef Kiesling übernahm jedoch die Sondergerichtsabteilung der Staatsanwaltschaft am Landgericht Wien, da er bereits zweimal einschlägig vorbestraft war, zudem zwischen seiner letzten Strafe und der nun angeklagten Tat nicht mehr als fünf Jahre verstrichen waren.

16 Die Definition des „Gewohnheitsverbrechers“ bzw. der „Gewohnheitsverbrecherin“" war zu finden in $\$ 20$ a RStGB, der am 4.9.1941 (rückdatiert vom 24.9.1941) im angeschlossenen Österreich in folgender Fassung Gültigkeit erhielt: „(1) Hat jemand durch eine mit bösem Vorsatz begangene gerichtlich strafbare Handlung eine Freiheitsstrafe verwirkt, nachdem er zweimal rechtskräftig wegen solcher strafbarer Handlungen zu Freiheitstrafe von je sechs Monaten oder zu strengeren Strafen verurteilt worden ist, und ergibt die Gesamtwürdigung der Taten, daß er ein gefährlicher Gewohnheitsverbrecher ist, so wird er, wenn die neue Tat an sich ein Vergehen oder eine Übertretung wäre, wegen Verbrechens mit Zuchthaus [...] bestraft." (RGB1. I, 1941, S. 581f.). Weitere Maßnahmen (wie die zeitlich unbegrenzte Sicherungsverwahrung) für sogenannte „Gewohnheitsverbrecher" und „Gewohnheitsverbrecherinnen“ wurden durch das „Gesetz gegen gefährliche Gewohnheitsverbrecher und über Maßnahmen zur Sicherung und Besserung" vom 24.11.1933 (RGBl. I, S. 995) im Reichsstrafgesetzbuch ( $\$ 42)$ festgeschrieben. $\$ 42$ erlangte in den Alpen- und Donaureichsgauen aber nie Gültigkeit.

17 Sämtliche Informationen zum Fall Josef Kiesling aus: WStLA, 2.3.13. Sondergericht, A1 - SHv Strafakten, Nr. 5572/47. 
Er erfüllte damit die Voraussetzungen, um als "Gewohnheitsverbrecher“ behandelt und vor das Sondergericht gestellt zu werden. Nachdem einer der Einbruchsversuche angeblich nach Beginn der Verdunkelung erfolgt war, wurde Kiesling zudem nicht nur nach $\$ 1$ der „Kriegswirtschaftsverordnung“, $\mathbb{S} \mathbb{S} 171 \mathrm{ff}$. des österreichischen Strafgesetzes $(\mathrm{StG})$ und als „Ge-

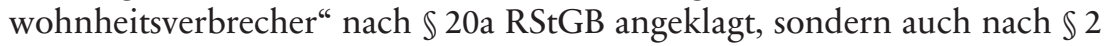
der „Volksschädlingsverordnung“ (RGBl. I, S. 1679).

Josef Kiesling und seinen Angehörigen war schon vor dem Vorliegen der Anklageschrift Ende Juni 1943 klar, dass die gerichtliche Verfolgung eine Frage von Leben und Tod bedeutete. Bereits im April wandte sich die Mutter Kieslings mit einem Gnadengesuch an Adolf Hitler beziehungsweise die Kanzlei des Führers. Sie betont darin ihre eigene Konformität und Hilfsbedürftigkeit, indem sie erwähnt, dass sie Trägerin des silbernen Mutterkreuzes, bereits 62 Jahre alt und krank sei. Sie verweist also auf die Erfüllung ihrer geschlechtsspezifischen Rolle, sowie ihren Körper beziehungsweise ihre schlechte Gesundheit. Nachdem sie dieses Bild der alten, kranken Mutter von sich gezeichnet hatte, bat sie darum, ihren Sohn an die Front zu schicken, sodass er kämpfend für das „deutsche Volk“ sterben könne, statt hinter Kerkermauern.

Währenddessen bemühte sich Josef Kiesling um das Wiedererlangen seiner Wehrwürdigkeit, die ihm aufgrund seiner Vorstrafen abgesprochen worden war. In einem diesbezüglichen Brief an den Untersuchungsrichter beschrieb er ein, wie er es nannte, „erschütterndes Bild“18 seiner Ehe und seiner wirtschaftlichen Lage, wobei er sich redlich bemüht habe, durch ehrliche und fleißige Arbeit seiner Frau und seinen drei Kindern ein Ernährer zu sein. Auch Kiesling versuchte also zu zeigen, dass er seine geschlechtsspezifische Rolle erfüllte und außerdem leistungsfähig und -willig war. Die Verantwortung für seine Taten übernahm er aber nicht: Das Eigeninteresse seiner Frau habe zur Anhäufung von Schulden und Pfändung des Großteils seines Eigentums geführt. Schließlich sei er gezwungen gewesen, verschiedenste Stellen anzunehmen, darunter auch Botengänge für den Erstangeklagten. Von dessen illegalen Geschäften oder von Diebstählen habe er keine Ahnung gehabt. Damit unterstellte Kiesling seiner Frau, was die Staatsanwaltschaft ihm unterstellte: eigennütziges, statt gemeinnütziges Verhalten.

18 Schreiben über Wiedererlangung der Wehrwürdigkeit, Josef Kiesling an Untersuchungsrichter i. Hause, 2.5.1943. WStLA, 2.3.13. Sondergericht, A1 - SHv Strafakten, Nr. 5572/47, Bl. 100f., Bl. 100. 
Für die Staatsanwaltschaft bestand jedoch kein Zweifel darüber, dass es Kiesling war, der als „gemeinschaftsuntauglich“ zu gelten hatte. Sie war überzeugt, dass er einen „eingewurzelten Hang zur Begehung von Verbrechen" aufwies. Schon in der Anklageschrift verlangte der Staatsanwalt da-

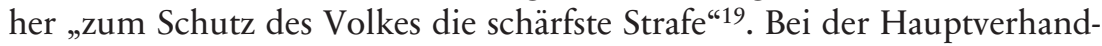
lung beantragte der Sitzungsvertreter die Todesstrafe. Das Sondergericht Wien unter Vorsitz von Landgerichtsdirektor Franz Werner folgte diesem Antrag und machte in der Urteilsbegründung deutlich, dass die Entscheidung eine Schutzmaßnahme für die Sicherheit der „Volksgemeinschaft“ darstellte:

„Das Sondergericht hat nach eingehender Würdigung des Vorlebens des Angeklagten, seiner Vorstrafen, der jetzigen Straftaten und seiner Gesamtpersönlichkeit gefunden, daß der Schutz der Volksgemeinschaft die Verhängung der Todesstrafe über den Angeklagten erfordert. Der Angeklagte verkörpert den Typ des antisozialen Menschen, des gefährlichen Gewohnheitsverbrechers, [...] in ausgeprägter Weise. Es ist nicht anzunehmen, daß auch die höchste zulässige zeitige Freiheitsstrafe von 15 Jahren der Volksgemeinschaft den Schutz bietet, den der Gesetzgeber ihr geben will, weil der Angeklagte [...] nach Verbüssung der Strafe erneut eine schwere Gefahr für die Volksgemeinschaft bilden würde. “20

In der Bewertung der Person Kieslings spielten die Differenzkategorien Geschlecht und Körper/Leistungsfähigkeit eine herausragende Rolle: Gerade weil Josef Kiesling vom Gericht als gesunder, junger Mann gesehen wurde, war es der Meinung, er hätte durchaus die Möglichkeit gehabt, sich „in einem ständigen Arbeitsverhältnis sozial zu bewähren“21, dass er dies nicht getan hatte, sei Zeichen seiner Unverbesserlichkeit. ${ }^{22}$ In seinem Gnadengesuch fasste Kiesling - wohl unter Anleitung seines Rechtsbeistands nochmals alle erlittenen Schicksalsschläge zusammen und versuchte, sein

19 Anklageschrift gegen Josef Kiesling, Oberstaatsanwalt (OStA) als Leiter der Anklagebehörde beim Landgericht (LG) Wien als Sondergericht (SG) an Vorsitzer des SG in Wien, 23.6.1943. WStLA, 2.3.13. Sondergericht, A1 - SHv Strafakten, Nr. 5572/47, Bl. 109f., Bl. 110.

20 Urteil (Entwurf) in Strafsache gegen Josef Kiesling, SG beim LG Wien, 4. Kammer, 30.7.1943. WStLA, 2.3.13. Sondergericht, A1 - SHv Strafakten, Nr. 5572/47, Bl. 122-129, Bl. 129.

21 Ebd.

22 Die dichotome Unterscheidung der Erziehbaren und Unverbesserlichen war seit Franz von Liszt eine der grundlegenden Differenzierungen im Strafrechtsdiskurs des deutschsprachigen Raums. Vgl. Stäcker 2012. 
Handeln als Folge einer Notlage und nicht seines Charakters zu erklären. Verzweifelt beteuert er, dass er ein nützliches Mitglied der „Volksgemeinschaft" und keineswegs antisozial sei. Er berichtet von zwei Begebenheiten, bei denen er kleinen Kindern das Leben gerettet habe. Er stellt seine Rolle als Versorger der Familie abermals in den Vordergrund, erinnert an seine eigenen Kinder und bittet um Milderung der harten Strafe. Das Gesuch wurde abgelehnt und Josef Kiesling im Alter von 35 Jahren am 23. September 1943 im Landgericht Wien hingerichtet.

Doch auch in Fällen, in denen nicht nach der „Volksschädlingsverordnung“ angeklagt und verurteilt wurde, war der Schutz der „Volksgemeinschaft" das handlungsleitende Motiv. So forderte auch im Fall des Wieners Emanuel Herdin ${ }^{23}$ der Sitzungsvertreter der Staatsanwaltschaft zum „Schutz der Volksgemeinschaft“ die Todesstrafe. Er wurde beschuldigt, Diebstähle begangen und Stoffe beiseitegeschafft zu haben, wobei er Personen bedroht und sich als Kriminalbeamter ausgegeben haben soll. Wie Josef Kiesling war auch Emanuel Herdin mehrfach (einschlägig) vorbestraft und galt der Staatsanwaltschaft als "gefährlicher Gewohnheitsverbrecher".

Bis zuletzt beteuerte Herdin, nicht der Haupttäter gewesen zu sein und belastete einen der Zeugen und Mittäter schwer. Der Staatsanwalt schenkte Herdin aber keinen Glauben, er attestierte diesem vielmehr eine starke „Verbrecherenergie“. Man erwartete sich von einer weiteren Freiheitsstrafe keinen Erfolg, „sodass der Schutz der Volksgemeinschaft und das Bedürfnis nach gerechter Sühne seine dauernde Ausschliessung aus der Volksgemeinschaft" 24 erfordern würde. Die endgültige Exklusion aus der „Volksgemeinschaft“ konnte nur eines bedeuten: den Tod. Das Sondergericht Wien folgte dem Antrag des Sitzungsvertreters der Staatsanwaltschaft in weiten Teilen und verhängte die Todesstrafe. ${ }^{25}$ In den Augen der urteilenden Richter würde Emanuel Herdin „,immer eine schwere und untrag-

23 Sämtliche Informationen zum Fall Emanuel Herdin aus: WStLA, 2.3.13. Sondergericht, A1 - SHv Strafakten, Nr. 6713/47.

24 Anklageschrift gegen Emanuel Herdin u.a., OStA als Leiter der Anklagebehörde beim LG Wien als SG an Vorsitzer des SG Wien, 14.11.1942. WStLA, 2.3.13. Sondergericht, A1 - SHv Strafakten, Nr. 6713/47, Bl. 363-374, Bl. 367.

25 Herdin wurde nach $\mathbb{} 1$ der „Kriegswirtschaftsverordnung“, $\mathbb{\$} \$ 185 \mathrm{f}$. StG, $\mathbb{9} 98 \mathrm{~b}$ StG, $\mathbb{1} 1$ der „Amtsanmaßungsverordnung“ und $\$ 20$ a RStGB in Verbindung mit $\$ 1$ des „Änderungsgesetzes“ schuldig gesprochen. Vgl. Urteil (Entwurf) in Strafsache gegen Emanuel Herdin u.a., SG beim LG Wien, 2. Kammer, 11.12.1942. WStLA, 2.3.13. Sondergericht, A1 - SHv Strafakten, Nr. 6713/47, Bl. 385-391. 
bare Bedrohung der Volksgemeinschaft bilden“, die „daher nur durch die Ausmerzung des Täters vor ihm wirksam geschützt werden“ 26 könne.

Der Verurteilte strebte erfolglos die Wiederaufnahme des Verfahrens an. Er unterstrich seinen Wert für die nationalsozialistische Gemeinschaft mit Hinweis auf sein stabiles Familienleben und seine Arbeitsfähigkeit und -willigkeit, indem er betonte, dass er inzwischen glücklich verheiratet und seit seiner letzten Haftentlassung 1941 ständig in Arbeit gestanden sei. Keiner seiner Dienstgeber habe ihm ein schlechtes Zeugnis ausgestellt. Er sei womöglich manchmal schwach und haltlos, aber kein „Volksschädling“, der dem „deutschen Volk“ gefährlich werden würde. Die Gnadenbehörde blieb anderer Meinung. Am 28. Jänner 1943 wurde Emanuel Herdin im Landgericht Wien enthauptet.

Josef Kiesling wie Emanuel Herdin waren in Wien geborene „Arier“, zum Zeitpunkt ihrer Verurteilung verheiratet und gingen als Hilfsarbeiter einer Beschäftigung nach. Beiden wurde vor allem ihr Vorstrafenregister zum Verhängnis, das in den Augen der entscheidenden Juristen von krimineller Neigung, also von einer angeborenen Minderwertigkeit, zeugte. Die Urteile lassen auch durchblicken, dass abgebrochene Lehren, längere Phasen der Erwerbslosigkeit oder der Hilfsarbeiterstatus negativ in die Personenbeurteilungen einflossen. Der körperliche und geistige Zustand findet in den Urteilen routinemäßig Erwähnung. Während er im Fall von Herdin nicht weiter von Bedeutung gewesen zu sein scheint, wurde Kiesling vorgeworfen, dass er fähig und gesund genug gewesen wäre, sich redlich fortzubringen. Selbstverständlich wurde auch die Zugehörigkeit zur NSDAP oder Parteiorganisationen überprüft. Die "Sozialität“ sowie Leistungsfähigkeit und „Leistungswilligkeit“ für die „Volksgemeinschaft“ und das NS-Regime wurden so ausgelotet und vom Gericht in seiner Gefahr-Nutzen-Abwägung berücksichtigt.

Dementsprechend bemüht waren Kiesling und Herdin vor und nach ihrer Verurteilung, ihre Leistungen für die "Volksgemeinschaft" zu betonen und sich als deren uneigennützige und vor allem besserungsfähige Mitglieder darzustellen. Beide unterstrichen ihr geschlechterrollenkonformes Verhalten als Ehemann und Familienvater sowie ihre Arbeitsleistung. Auch die Bemühungen Kieslings in den Wehrdienst einzutreten - eine Art

26 Urteil (Entwurf) in Strafsache gegen Emanuel Herdin u.a., SG beim LG Wien, 2. Kammer, 11.12.1942. WStLA, 2.3.13. Sondergericht, A1 - SHv Strafakten, Nr. 6713/47, Bl. 385-391, B1. 390. 
„Sühne“, ${ }^{27}$ die Personen nicht-deutscher Herkunft verwehrt war - sollten von seiner Opferbereitschaft für das „Volk“ zeugen.

Gerichtlich Verfolgte beziehungsweise deren Rechtsbeistände konnten die „Volksgemeinschaft“ als Referenzobjekt des Sicherheitsdiskurses auch sehr direkt in die Argumentation einbringen, auch wenn es sich nicht um "arische" Beschuldigte handelte. Der Anwalt von Panagiotis Mangos ${ }^{28}$ beispielsweise versuchte, dem zum Tode verurteilten Griechen damit das Leben zu retten:

Der geschiedene Mangos war 1942 im Alter von 25 Jahren mit einem Arbeitertransport angeblich freiwillig nach Wien gekommen, wo er am Bahnhof Stadlau als Hilfsarbeiter der Reichsbahn eingesetzt wurde. Dabei soll er zahlreiche Vorstrafen absichtlich verheimlicht haben. Anfang Mai 1943 floh Mangos aus dem Arbeitslager, in dem er untergebracht war, und war seither wohnungslos. Während seiner Zeit in Wien soll er an mindestens fünf Diebstählen führend beteiligt gewesen sein, im Zuge derer während der Verdunkelung bezugsscheinpflichtige Waren sowie Lebensmittelkarten entwendet worden waren. Die Staatsanwaltschaft klagte ihn nach $\$ 1$ der „Kriegswirtschaftsverordnung“, $\$ 2$ der „Volksschädlingsverordnung“, $\mathbb{\$} \$ 171 \mathrm{ff}$. StG und $\mathbb{2} 20 \mathrm{a}$ RStGB (als „Volksschädling“ und „Gewohnheitsverbrecher") an. Neben der Ausnutzung der Verdunkelung war auch die angebliche „Arbeitsscheu“ des Angeklagten ausschlaggebend für dessen Klassifizierung als „Volksschädling“. Auf Weisung des Reichsministeriums der Justiz beantragte der Sitzungsvertreter die Todesstrafe, die das Sondergericht Wien am 6. November 1943 verhängte. Die Richter waren der Überzeugung, dass die „völlige Beseitigung dieses Angeklagten“ nötig sei „zur Wahrung von Ordnung und Sicherheit und sohin zum Schutz der Volksgemeinschaft“. ${ }^{29}$ Auch der „Abschreckungsgesichtspunkt“ gegenüber anderen ausländischen Arbeitskräften spielte bei der Urteilsfindung eine bedeutende Rolle. Dass Panagiotis Mangos ein umfangreiches Geständnis abgelegt hatte und Reue zeigte - üblicherweise als strafmildernd anerkannte Umstände -, konnte deshalb nach Ansicht des Gerichts nicht ins Ge-

27 Die Sühne des begangenen Unrechts ist ein traditioneller Straf(vollzugs)zweck, der im NS-Regime wieder stärker an Bedeutung gewann, nachdem er in der Weimarer Republik hinter die Resozialisierung zurücktrat. Daneben legten die Nationalsozialisten vor allem Wert auf die Abschreckungswirkung der Strafe.

28 Sämtliche Informationen zum Fall Panagiotis Mangos aus: WStLA, 2.3.13. Sondergericht, A1 - SHv Strafakten, Nr. 7289/47.

29 Urteil (Entwurf) in Strafsache gegen Joanis Kastrenelis u.a., SG beim LG Wien, 1. Kammer, 6.11.1943. WStLA, 2.3.13. Sondergericht, A1 - SHv Strafakten, Nr. 7289/47, Bl. 98-109, Bl. $107 f$. 
wicht fallen. Die ausländische Herkunft des Angeklagten und die Prävention weiterer Straftaten ausländischer (Zwangs-)Arbeitskräfte waren hier offensichtlich von größerer Relevanz.

Im Gnadengesuch argumentiert der Rechtsbeistand Mangos', dass der Verurteilte mit den Diebstählen hauptsächlich seine eigenen Landsleute geschädigt habe und dass die „deutsche Volksgemeinschaft“ durch seine Abschiebung nach Griechenland weiterhin von ihm verschont bleiben könne. Das Exil sei eine angemessene und ausreichende Schutzmaßnahme für die „Volksgemeinschaft“. Diese Argumentation überzeugte weder den zuständigen Vorsitzenden des Sondergerichts Wien noch die Gnadenbehörde. Das Gnadengesuch wurde abgelehnt.

Es wurde jedoch nicht unbedingt jede als "Gewohnheitsverbrecherin“ und jeder als "Gewohnheitsverbrecher" Angeklagte und Verurteilte vom Sondergericht Wien als extrem gefährlich und zu „beseitigen“ eingestuft. Selbst wenn die betreffende Person nicht-deutscher Herkunft war und zahlreiche Vorstrafen vorlagen, konnten die Richter des Sondergerichts Wien eine zeitlich begrenzte Freiheitsstrafe zur angemessenen Schutzmaßnahme erklären - auch gegen den Widerstand der Staatsanwaltschaft. Die Staatsanwaltschaft und das Sondergericht Wien stimmten in der Beurteilung der Gefährlichkeit einer Person nicht immer überein. So wurde etwa das Urteil des Sondergerichts Wien vom 6. Mai 1944 gegen Luka und Lucija Živković (hierbei handelt es sich um Pseudonyme) ${ }^{30}$ nach einer von der Staatsanwaltschaft des Landgerichts Wien angeregten Nichtigkeitsbeschwerde des Oberreichsanwalts aufgehoben und der Fall am 30. August 1944 ein zweites Mal verhandelt.

Luka Živković war mit vier weiteren Personen wegen Diebstahls ${ }^{31}$ als „Volksschädling“ und „gefährlicher Gewohnheitsverbrecher" angeklagt worden. Seine unbescholtene Ehefrau Lucija Živković soll einen Teil des Diebesguts verhehlt haben. Das Sondergericht Wien sprach sie in allen Punkten frei, während es den mehrfach vorbestraften Luka Živković aufgrund dreier Taschendiebstähle zu sechs Jahren Zuchthaus verurteilte. Die

30 Sämtliche Informationen zum Fall Živković (Pseudonym) aus: WStLA, 2.3.13. Sondergericht, A1 - SHv Strafakten, Nr. 9350/47.

31 Nach $\mathbb{} 1$ der „Kriegswirtschaftsverordnung“, $\mathbb{S} \$ 2$ und 4 der „Volksschädlingsverordnung“, $\mathbb{S} 171 \mathrm{ff}$. StG und $\$ 20$ a Abs. II RStGB in Verbindung mit $\$ 1$ des „Änderungsgesetzes“. Vgl. Anklageschrift gegen Juliana Marković u.a., OStA als Leiter der Anklagebehörde beim LG Wien als SG an Vorsitzer des SG Wien, April 1944. WStLA, 2.3.13. Sondergericht, A1 - SHv Strafakten, Nr. 9350/47, Bl. 252257. 
Staatsanwaltschaft hatte allerdings die Todesstrafe gefordert. Unzufrieden mit dem Urteil, strengte sie erfolgreich die Wiederaufnahme an.

Der Freispruch von Lucija Živković wurde wiederholt und Luka Živkovićs Strafe von sechs auf sieben Jahre Zuchthaus erhöht. Nach wie vor konnte man ihm nicht mehr als die drei Taschendiebstähle beweisen. Živković war, nach Ansicht der Richter, mit den übrigen Angeklagten nicht „bandenmäßig“ organisiert gewesen. Obwohl er den Charakter eines Volksschädlings habe und auch ein „eingewurzelter Hang zum Diebstahl“32 vorhanden sei, wollte das Sondergericht das „Änderungsgesetz“33 nicht anwenden. Die Straftaten wären nämlich nicht so gravierend, dass sie die „Ausmerzung des Täters verlangen würde“34. In den Augen des Gerichts war Živković nur von „durchschnittlicher Gefährlichkeit“ und eine empfindliche Freiheitsstrafe daher eine ausreichende Maßnahme zum „Schutz der Volksgemeinschaft“.

Als strafmildernder Umstand wurde die Sorgepflicht für seine Frau und die beiden gemeinsamen Kinder - also die geschlechtsspezifische Rolle als Versorger - berücksichtigt. Auf die nicht-deutsche Herkunft und seine Arbeitsleistung oder -fähigkeit wurde in der Urteilsbegründung nicht eingegangen, was bei Vorliegen eines langen Vorstrafenregisters unüblich war. Gegen Luka Živković lagen mehrere Vorstrafen wegen Diebstahls und weitere 22 Vorstrafen wegen Vagabundierens vor.

Das Gericht sah auch keinen Anlass, andere Punkte aus Živkovićs ansonsten relativ unauffälligem Lebenslauf weiter zu beleuchtet: Der zum Zeitpunkt seiner Verurteilung 32-jährige Serbe hatte nach dem Besuch von vier Klassen Volksschule eine Kellnerlehre abgeschlossen und diesen Beruf jahrelang ausgeübt. In dieser Zeit heiratete er, die Ehe galt als glücklich. 1941 eröffnete Živković ein Gasthaus, das er aber im selben Jahr aus wirtschaftlichen Gründen wieder schließen musste. Ende Juli 1942 kam er nach Wien und fand bei einer Holzhandlung am Nussdorfer Platz als Hilfsarbeiter Beschäftigung. Diese Arbeit soll er wenige Wochen vor seiner Verhaftung niedergelegt haben.

Die Strafe wurde bei der Wiederverhandlung auf sieben Jahre Zuchthaus erhöht, nachdem festgestellt worden war, dass Živković spielte. Diese schlechte Angewohnheit zog das Sondergericht unter Vorsitz des Land-

32 Urteil in Strafsache gegen Luka Živković u.a., SG beim LG Wien, 2. Kammer, 30.8.1944. WStLA, 2.3.13. Sondergericht, A1 - SHv Strafakten, Nr. 9350/47, o.P.

$33 \rrbracket 1$ des „Änderungsgesetzes“ von 1941 schrieb zum „Schutz der Volksgemeinschaft" die Todesstrafe vor. Siehe FN 7.

34 Urteil in Strafsache gegen Luka Živković u.a., SG beim LG Wien, 2. Kammer, 30.8.1944. WStLA, 2.3.13. Sondergericht, A1 - SHv Strafakten, Nr. 9350/47, o.P. 
gerichtsdirektors Dr. Otto Eder schließlich als Argument heran, um die Strafe zu verschärfen und den involvierten Staatsanwälten etwas entgegenzukommen.

Die Sondergerichte im Oberlandesgerichtsbezirk Wien wichen in ihren Urteilen von den Anträgen der Staatsanwaltschaft meist nur minimal ab. Nur etwa in einem Fünftel aller Heimtücke- und Kriegswirtschaftsverfahren ist ein auffälliger Unterschied festzustellen. ${ }^{35}$ Dass sich das Sondergericht Wien in diesem Fall selbst bei der Wiederverhandlung im August 1944 nicht zu einer bedeutend härteren Strafe bewegen ließ, zeugt zum einen von der Agenda der Staatsanwaltschaft, zum anderen vom Unwillen der 2. Kammer des Sondergerichts Wien unter Eders Vorsitz, ihr eigenes Urteil zu revidieren. Dies hatte zur Folge, dass sich das Gericht weigerte, einen harmlosen Kleinkriminellen als Gefahr für die „Volksgemeinschaft“ hinrichten zu lassen. ${ }^{36}$

Doch auch Landgerichtsrat Eder sprach zahlreiche Todesurteile aus: 1944 ließ er zumindest 25 Personen hinrichten, ${ }^{37}$ darunter auch drei Mitangeklagte des Ehepaars Živković. Diese hätten, anders als der gelegentlich rückfällig werdende Živković, zahlreiche gemeinsam geplante Diebstähle begangen und dabei wertvolle Gegenstände entwendet. Dadurch hätten sie als Ausländer und Ausländerinnen die „Gastfreundschaft" des Deutschen Reiches missbraucht, was bei der Straffindung erschwerend ins Gewicht fiel. Dass sie die Taten als Nicht-Deutsche begangen hatten, wurde in der Urteilsbegründung dezidiert festgehalten:

„Die große Anzahl der Diebstähle, die Höhe und vor allem die Empfindlichkeit des angerichteten Schadens und die schamlose Frechheit, mit der sie als Ausländer im Reiche die öffentliche Sicherheit gestört

35 Eigene statistische Auswertung, basierend auf 415 vor den Sondergerichten des Oberlandesgerichtsbezirks Wien (Krems, St. Pölten, Wien und Znaim) verhandelten Kriegswirtschafts- und Heimtückeverfahren.

36 Was nicht bedeutete, dass die Verurteilten damit dem Tod entronnen wären. Von den Lebensbedingungen hinter Gittern abgesehen, wurden zahlreiche Bestrafte während oder nach der Verbüßung ihrer Strafhaft an die Gestapo oder SS überstellt, damit diese weitere „Schutzmaßnahmen“ treffen konnte, wie es die Gestapo formulierte. Auch im Fall Živković hatte die Gestapo zu diesem Zweck eine Rücküberstellung beantragt. Vgl. Schreiben über Andjelkovic Blagoje u.a., KripoLeitstelle Wien an Leiter der Anklagebehörde beim LG Wien als SG, 7.8.1943. WStLA, 2.3.13. Sondergericht, A1 - SHv Strafakten, Nr. 9350/47, Bl. $165 f$.

37 WStLA, 2.3.13. Sondergericht, A2 - Anklagschriften, Todesurteile und Diverses, Karton 3, Mappe A2 - Anklageschriften SJs 1944-1945. 
haben, lassen ihre Taten derart verwerflich erscheinen, daß die gerechte Sühne die Beseitigung solcher Menschen verlangt. " ${ }^{38}$

Die Formulierung lässt darauf schließen, dass bei der Bewertung der drei Angeklagten zuvorderst der Tatumfang und die Differenzkategorie „Rasse“/,Volkstum“ eine Rolle spielte.

Unter diesen Angeklagten war auch Juliana Marković, eine im damals königlichen Ungarn geborene unbescholtene Schneiderin. Die Serbin war, wie Luka Živković, verheiratet und hatte zwei Kinder. Sie war im April 1943 nach Wien gekommen, wo sie, wie sie aussagte, wegen einer Erkrankung für arbeitsunfähig befunden worden sei. Offenbar konnten Juliana Marković zahlreiche Taschendiebstähle nachgewiesen werden. Sie bestritt jedoch bei ihren Verhören und noch während der Hauptverhandlung jegliche Beteiligung. Auch dies bewertete das Gericht als „Frechheit“. Die Verärgerung der Richter dürfte mit ein Grund dafür gewesen sein, dass Marković ungeachtet ihres bisher nicht zu beanstandenden Lebenswandels zum Tode verurteilt wurde. Da die formalen Voraussetzungen nach $\$ 20 \mathrm{a}$ RStGB Abs. 1 nicht erfüllt waren, musste man sich mit Abs. 2 behelfen, ${ }^{39}$ um sie als "gefährliche Gewohnheitsverbrecherin“ einstufen und so zum Tode verurteilen zu können. ${ }^{40}$

Wie oben gezeigt, fiel erschwerend ihre nicht-deutsche Herkunft ins Gewicht. Hingegen sah man in ihrer Unbescholtenheit oder ihrem kränklichen Gesundheitszustand - Umstände, die vom Sondergericht Wien in vielen anderen Fällen als strafmildernd anerkannt wurden - keinen Grund, sie als ungefährlicher für die öffentliche Sicherheit einzuschätzen als ihre männlichen, vorbestraften Komplizen. Auch Marković' Geschlecht und ihre zwei unmündigen Kinder waren in den Augen des Gerichts kein Grund, sie zumindest vor der Todesstrafe zu verschonen. Stattdessen

38 Urteil in Strafsache gegen Juliana Marković u.a., SG beim LG Wien, 2. Kammer, 6.5.1944. WStLA, 2.3.13. Sondergericht, A1 - SHv Strafakten, Nr. 9350/47, Bl. 290-304, Bl. 303.

39 Abs. 2 besagte: „Dieselbe Verschärfung ist zulässig, wenn der Täter, auch ohne zweimal rechtskräftig verurteilt worden zu sein, noch mindestens zwei gerichtlich strafbare Taten mit bösem Vorsatz begangen hat und die Gesamtwürdigung der Taten ergibt, daß er ein gefährlicher Gewohnheitsverbrecher ist." (Abs. 1 siehe FN 16)

40 Sie wurde nach $\mathbb{1} 1$ der „Kriegswirtschaftsverordnung“, $\mathbb{S} 171 \mathrm{ff}$. StG, $\mathbb{S} 2$ und 4 der „Volksschädlingsverordnung“ und nach $\mathbb{2} 20$ a RStGB in Verbindung mit $\mathbb{1} 1$ Abs. 2 des „Änderungsgesetzes“ verurteilt. Urteil in Strafsache gegen Juliana Marković u.a., SG beim LG Wien, 2. Kammer, 6.5.1944. WStLA, 2.3.13. Sondergericht, A1 - SHv Strafakten, Nr. 9350/47, B1. 290-304. 
nutzte das Sondergericht Wien die Möglichkeit, sie als gefährlich für die „Volksgemeinschaft“ hinrichten zu lassen. Es kann angenommen werden, dass das wiederholte Leugnen Marković in Verbindung mit ihrem Geschlecht das gängige Bild des renitenten, aufsässigen Weibsbilds vor den Augen der Richter heraufbeschwor. Hätte sie mehr dem Klischee der weichen, gefügigen Frau entsprochen, hätte ihr das womöglich das Leben gerettet.

\section{Fazit}

Der NS-Justizapparat ist als zentrales Sicherheitsorgan zu benennen, dessen Aufgabe auch der Schutz vor Gefahren durch die Ausschaltung von Bedrohung war. Die Versicherheitlichungsprozesse, an denen juristische Akteure beteiligt waren, waren so grundlegend, dass sie mit der Neudefinition krimineller Handlungen und Personen - als existenzielle Bedrohungen der "Sicherheit" und „Gesundheit" der nationalsozialistischen Gemeinschaft - die Grenze zwischen dem, was als "normal“ galt und was nicht, verschoben. Die Rolle der Juristen im NS-Regime muss daher als extreme Form der Involvierung in Versicherheitlichungsprozesse bezeichnet werden. Sie beteiligten sich aktiv daran, die papierenen Bedrohungsszenarien der nationalsozialistischen Ideologie auszuformulieren und - mit realen Maßnahmen - Wirklichkeit werden zu lassen. Dabei urteilten sie nicht nur über das Tatsächliche, sondern auch über das Mögliche: Die Strafrechtspraxis bezog sich auf in der Zukunft liegende Bedrohungsszenarien.

Diese projektive Versicherheitlichungstechnik richtete sich vor allem gegen "Gewohnheitsverbrecher" und „Gewohnheitsverbrecherinnen“, die als multidimensionales Sicherheitsproblem definiert wurden. Sie waren durch die „Überhöhung und moralisierende Betrachtung von Delinquenz“ (Roth 2008, S. 41) nicht mehr nur kriminell, sondern unverbesserliche Feinde der Gemeinschaft des „deutschen Volkes“. Die als „rassisch rein“ und gesund imaginierte „Volksgemeinschaft“ war der Referenzpunkt der Schutz- und Sicherheitsargumente. Die Exklusion aus oder Fernhaltung von dieser wurde zu einer vordringlichen Sicherheitsmaßnahme.

Bei der Bewertung der Gefährlichkeit der Einzelnen kam es auf das komplexe Zusammenspiel von tat- und personenbezogenen Kriterien an. In den Prozessakten findet man immer wieder Argumente, die sich auf die gängigen Differenzkategorien beziehen: Geschlecht, Körper/Leistungsfähigkeit und „Rasse“/,Volkstum“ nahmen situativ einen unterschiedlichen Stellenwert ein. Das Gericht zog diese Argumente als Kriterien heran, 
um die „Sozialität“ oder „Gemeinschaftsfähigkeit" zu bewerten und so in Verbindung mit dem kriminalisierten Verhalten den Wert und die (zukünftige) Gefährlichkeit einer Person festzustellen. Dabei musste, wie im Fall Živković, zwischen den Staatsanwälten und Richtern nicht zwingend Einigkeit über das Gefahrenpotenzial bestehen.

In dem Wissen, dass das geschlechterrollenkonforme Verhalten oder die Leistungsfähigkeit sich positiv auf die Abwägung von Nutzen und Gefährlichkeit einer Person auswirken konnte, brachten die Angeklagten bzw. deren Rechtsbeistände diese Kriterien oftmals selbst in die Diskussion ein. Die (Gnaden-)Gesuche zeugen von der Intention, die Beschuldigten als möglichst gesellschaftskonform und opferbereit zu beschreiben, so dass sie der angedrohten, harten Strafe entgehen konnten.

In den Fällen der „Volkgenossen“ Kiesling und Herdin sind in erster Linie das Geschlecht und der Körper/die Leistungsfähigkeit als Argumente in den Akten präsent. Die Angeklagten versuchten ihre Rolle als Versorger der Familie sowie ihren Arbeits- und Opferwillen zu betonen. In den Fällen nicht-deutscher Angeklagter scheinen diese Kriterien weniger von Bedeutung gewesen zu sein, hier stand vor allem die ausländische Herkunft im Vordergrund. Sie konnte, wie im Fall Marković und auch Mangos, musste aber nicht, wie der Fall Živković zeigt, strafverschärfend wirken.

Was das Verfahren gegen Marković aber vor allem zeigt, ist, dass die Verteidigungsstrategie bzw. die Kooperationsbereitschaft mit dem Gericht neben dem Tatumfang eine maßgebliche Rolle bei der Urteilsfindung spielte. Und auch die Verteidigungsstrategie dürfte nicht unabhängig von Differenzkategorien wie dem Geschlecht beurteilt worden sein.

Das Zusammenspiel all dieser Urteilskriterien sowie die getroffenen Entscheidungen waren hochgradig situativ. Festgestellt werden kann aber, dass, wenn eine Person einmal als gefährlich für die öffentliche Sicherheit eingestuft worden war, ihre drakonische Bestrafung erwartet werden konnte. Ein Sicherheitsproblem darzustellen, bedeutete für bisherige „Volksgenossen" und „Volksgenossinnen“ genauso wie für die „Fremdvölkischen" nicht selten den Tod. 


\section{Quellen und Literatur}

\section{Archive}

Wiener Stadt- und Landesarchiv (WStLA), Bestand 2.3.13 Sondergericht: A1 - SHv Strafakten (Nr. 5572/47, Nr. 6713/47, Nr. 7289/47, Nr. 9350/47) A2 - Anklagschriften, Todesurteile und Diverses

\section{Gesetze und Verordnungen}

Gesetz gegen gefährliche Gewohnheitsverbrecher und über Maßnahmen zur Sicherung und Besserung vom 24.11.1933. RGB1. I, S. 995.

Gesetz gegen heimtückische Angriffe auf Staat und Partei und zum Schutz der Parteiuniformen vom 20.12.1934. RGBl. I, S. 1269.

Gesetz zur Änderung des Strafgesetzbuchs vom 28.6.1935. RGBl. I, S. 839-843.

Verordnung über die Erweiterung der Zuständigkeiten der Sondergerichte vom 20.11.1938. GBlÖ. Nr. 614/1938.

Kriegswirtschaftsverordnung vom 4.9.1939. RGBl. I, S. 1609-1613.

Verordnung gegen Volksschädlinge vom 5.9.1939. RGBl. I, S. 1679.

Verordnung über die Zuständigkeit der Strafgerichte, die Sondergerichte und sonstige strafverfahrensrechtliche Vorschriften vom 21.2.1940. RGBl. I, S. 405-411.

Gesetz zur Änderung des Reichstrafgesetzbuches vom 4.9.1941. RGBl. I, S. 549f.

Verordnung zur Durchführung des Gesetzes zur Änderung des Reichsstrafgesetzbuchs vom 24.9.1941. RGBl. I, S. 581f.

\section{Sekundärliteratur}

Bajohr, Frank/Wildt, Michael (Hrsg.) (2009): Volksgemeinschaft. Neue Forschungen zur Gesellschaft des Nationalsozialismus. Frankfurt a. M.: Fischer Taschenbuch Verlag.

Balzacq, Thierry (Hrsg.) (2010): Securitization Theory. How Security Problems Emerge and Dissolve. London: Routledge.

Conze, Eckart (2018): Geschichte der Sicherheit. Entwicklung - Themen - Perspektiven. Göttingen: Vandenhoeck \& Ruprecht.

Hörath, Julia (2017): „Asoziale“ und „Berufsverbrecher“ in den Konzentrationslagern 1933 bis 1938. Göttingen: Vandenhoeck \& Ruprecht.

Kranebitter, Andreas (2018): Der „Kampf gegen das Verbrechertum“ im nationalsozialistischen Österreich. Die Kriminalpolizei und die Radikalisierung der NSVerfolgungspolitik nach 1938. In: Österreichische Zeitschrift für Geschichtswissenschaften 29, H. 1, S. 148-179. 
Krasmann, Susanne/Kreissl, Reinhard/Kühne, Sylvia/Paul, Bettina/Schlepper, Christina (2014): Die gesellschaftliche Konstruktion von Sicherheit. Zur medialen Vermittlung und Wahrnehmung der Terrorismusbekämpfung. Berlin: Freie Universität Berlin.

Kunze, Thomas (2005): Der Sicherheitsdiskurs. Die Innere Sicherheitspolitik und ihre Kritik. Bielefeld: transcript Verlag.

Landwehr, Achim (2008): „Gute Policey“. Zur Permanenz der Ausnahme. In: Lüdtke, Alf/Wildt, Michael (Hrsg.): Staats-Gewalt: Ausnahmezustand und Sicherheitsregimes. Historische Perspektiven. Göttingen: Wallstein Verlag, S. 3963.

Reusch, Marius Sebastian/Paz, Reut Yeal/Tebruck, Stefan (2018): Juristen als „Sicherheitsakteure“. Eine Einführung. In: Westermeier, Carola/Carl, Horst (Hrsg.): Sicherheitsakteure. Epochenübergreifende Perspektiven zu Praxisformen und Versicherheitlichung. Baden-Baden: Nomos, S. 95-110.

Roth, Thomas (2008): Kriminalpolitik im NS-System. In: Hans-Jürgen Lange (Hrsg.), Kriminalpolitik. Wiesbaden: VS Verlag für Sozialwissenschaften, S. 3755.

Schmitt, Carl (2015): Politische Theologie. Vier Kapitel zur Lehre von der Souveränität. 10. Aufl., Berlin: Duncker \& Humblot.

Stäcker, Therese (2012): Die Franz von Liszt-Schule und ihre Auswirkungen auf die deutsche Strafrechtsentwicklung. Baden-Baden: Nomos.

Wagner-Kern, Michael (2016): Präventive Sicherheitsordnung. Zur Historisierung der Sicherungsverwahrung. 2., überarb. Aufl., Berlin: Berliner WissenschaftsVerlag.

Wedrac, Stefan (2017): Die politische Einstellung der Richter des Präsidiums und des Geschäftsbereiches Zivil- und Handelssachen des Landgerichts Wien 1942. Versuch einer Kollektivbiographie. In: Beiträge zur Rechtsgeschichte Österreichs, 7. Jg., Bd. 2, S. 252-268.

Westermeier, Carola/Carl, Horst (2018): Einleitung: „Sicherheitsakteure“. In: Dies. (Hrsg.): Sicherheitsakteure. Epochenübergreifende Perspektiven zu Praxisformen und Versicherheitlichung. Baden-Baden: Nomos, S. 11-27.

Wetzell, Richard F. (2000): Inventing the Criminal. A History of German Criminology, 1880-1945. Chapel Hill und London: The University of North Carolina Press.

Winker, Gabriele/Degele, Nina (2010): Intersektionalität. Zur Analyse sozialer Ungleichheiten. Bielefeld: transcript Verlag. 